\title{
GMR
}

\section{Analysis of drug-resistant gene detection of blaOXA-like genes from Acinetobacter baumannii}

\author{
D.K. Yang, H.J. Liang, H.L. Gao, X.W. Wang and Y. Wang \\ Department of Infections, The First Affiliated Hospital of Xinxiang Medical University, \\ Weihui, China \\ Corresponding author: D.K. Yang \\ E-mail: yangdk_xmu@163.com \\ Genet. Mol. Res. 14 (4): 18999-19004 (2015) \\ Received August 7, 2015 \\ Accepted October 7, 2015 \\ Published December 29, 2015 \\ DOI http://dx.doi.org/10.4238/2015.December.29.7
}

\begin{abstract}
Our study determines the resistance gene profile of a set of Acinetobacter baumannii hospital isolates. A. baumannii is responsible for nosocomial outbreaks and sporadic infections. We extracted and PCR amplified bacterial DNA isolated from patients with ages below 60 years $(23.36 \%)$ and above 60 years $(76.64 \%)$. Most of the patients were admitted in the ICU (36.13\%) and pneumology departments $(28.47 \%)$. Of 164 isolated strains, $16(9.75 \%)$ contained OXA-51, 8 $(4.88 \%)$ contained OXA-58, and $140(85.37 \%)$ contained both OXA51 and OXA-23. Additionally, $8(7.41 \%)$ strains containing OXA-58 and $100(92.59 \%)$ strains containing both OXA-51 and OXA-23 showed multidrug-resistance. Drug resistance rates of $A$. baumannii to amikacin, tobramycin-levofloxacin, and cotrimoxazole were above $90 \%$, while drug resistance rates to ampicillin, cefotetan, cefazolin, cefoperazone, and nitrofurantoin were $100 \%$. In conclusion, we found that isolated strains containing OXA-51 and OXA-23 were more likely to be resistant or have decreased sensitivity to carbapenems.
\end{abstract}

Key words: Acinetobacter baumannii; Drug-resistant gene; OXA-51; OXA-23; OXA-24; OXA-58 


\section{INTRODUCTION}

Acinetobacter baumannii, an aerobic non-motile gram-negative coccobacillus, is an opportunistic pathogen, which is widely found in intensive care units (ICUs), and induces nosocomial infections such as pneumonia, septicemia, and urinary tract and wound infections. $A$. baumannii is frequently involved in outbreaks and can persist in the environment for several days (Metan et al., 2013). Previous studies reported that $A$. baumannii is the most common pathogenic bacteria isolated from hospitalized patients with pneumonia (Cefai et al., 1990; Lin et al., 2010). A. baumannii is usually found to be resistant to many drugs, including third-generation cephalosporins, aminoglycosides, and fluoroquinolone (Sinha and Srinivasa, 2007).

Multidrug-resistant $A$. baumannii strains can acquire antibiotic-resistance genes through class 1 integrons that carry single or multiple gene cassettes (Petersen et al., 2000). Integrons are genetic elements encoding antibiotic resistance genes that can integrate or mobilize their gene cassettes (Recchia and Hall, 1995). Aminoglycoside resistance genes, which are class 1 integrons, play an important role in the enzymatic inactivation of aminoglycoside antibiotics and could cause

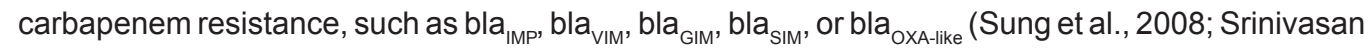
et al., 2009). Carbapenemase production is the most well described mechanism of resistance to carbapenems (Poirel et al., 2007). The most common mechanism of drug resistance is associated with hydrolyzing $\beta$-lactamases of metallo- $\beta$-lactamases (Ambler class B) and oxacillinases (Ambler class D). In our study, we collected 131 strains to detect drug-resistance genes by PCR analysis and conducted homology analysis. We determined the resistance gene profile of $A$. baumannii responsible for nosocomial outbreaks and sporadic infections.

\section{MATERIAL AND METHODS}

\section{Patients}

In total, 274 A. baumannii isolates were collected from the First Affiliated Hospital of Xinxiang Medical University between May 2012 and May 2014. Strains were identified as $A$. baumannii by a PCR test using two amplification bands. We used 164 strains of $A$. baumannii to detect the distribution of genes.

\section{Bacterial isolates and gene analysis}

The DNA of bacteria was extracted using the TIANamp Bacteria DNA Kit (Tiangen Biotech Co., Ltd., Beijing, China) and amplified using Taq PCR Master Mix (Shanghai Lifefeng Biotech Co., Ltd, Shanghai, China). Primers for OXA-51, OXA-23, OXA-24, and OXA-58 were produced by Shanghai Jierui Biological Engineering Co., Ltd. The primers for OXA-51, OXA-23, OXA-24, and OXA-58 were as follows: for OXA-51, TAATGCTTTGATCGGCCTTG (forward) and TGGATTGCACTTCATCTTGG (reverse); for OXA-23, ATGAATAAATATTTTACTTG (forward) and TTAAATAATATTCAGCTGTT (reverse); for OXA-24, TTCCCCTAACATGAATTTGT (forward) and GTACTAATCAAAGTTGTGAA (reverse); and for OXA-58, TTATCAAAATCCAATCGGC (forward) and TAACCTCAAACTTCTAATTC (reverse).

Each PCR mix was comprised of $25 \mu \mathrm{L}$ Taq Mix, $1 \mu \mathrm{L}$ primers, $1 \mu \mathrm{L}$ template, and $50 \mu \mathrm{L}$ RNase-Free $\mathrm{dH}_{2} \mathrm{O}$. The internal reference for OXA-51, OXA-23, OXA-24, and OXA-58 was OXA- 
51. The PCR amplification was as follows: 5 min initial denaturation at $94^{\circ} \mathrm{C}$, followed by 30 cycles of denaturation for $30 \mathrm{~s}$ at $95^{\circ} \mathrm{C}$, annealing for $90 \mathrm{~s}$ at $72^{\circ} \mathrm{C}$, and extension for $30 \mathrm{~s}$ at $72^{\circ} \mathrm{C}$, followed by a final extension for $5 \mathrm{~min}$ at $72^{\circ} \mathrm{C}$. PCR products were verified by gel electrophoresis using a $1.5 \%$ agarose gel and visualized using ethidium bromide staining. The 353-, 501-, 1024-, and 507-bp amplicons represented the OXA-51, OXA-23, OXA-24, and OXA-58 genes, respectively.

\section{Statistical analysis}

Frequencies were used to describe the distribution of categorical variables. Median and interquartile range was used for continuous variables. The association between $A$. baumannii and drug resistance was analyzed by the chi-square test. All $P$ values were two sided, and $P<0.05$ was considered to be statistically significant.

\section{RESULTS}

Characteristics of 274 patients are shown in Table 1. Ages of 64 patients $(23.36 \%)$ were below 60 years, while those of the remaining 210 patients $(76.64 \%)$ were above 60 years. Most of the patients were in the ICU $(36.13 \%)$ or pneumology departments $(28.47)$. Of 164 strains of A. baumannii, 139 (84.76\%) were clinical specimens isolated from sputum and $25(15.24 \%)$ were isolated from cerebrospinal fluid, catheters, or pleural effusion.

\begin{tabular}{|c|c|c|}
\hline Variables & $N=274$ & $\%$ \\
\hline \multicolumn{3}{|l|}{ Age (years) } \\
\hline$<60$ & 64 & 23.36 \\
\hline$\geq 60$ & 210 & 76.64 \\
\hline \multicolumn{3}{|c|}{ Location of Acinetobacter baumannii } \\
\hline ICU & 99 & 36.13 \\
\hline Emergency & 53 & 19.34 \\
\hline Cerebral surgery & 44 & 16.06 \\
\hline Pneumology & 78 & 28.47 \\
\hline
\end{tabular}

Strains containing the drug resistant genes OXA-51, OXA-23, OXA-24, and OXA-58 are shown in Table 2 and Figure 1. Of 164 isolated strains, 16 (9.75\%) contained OXA-51, 8 (4.88\%) contained OXA-58, and 140 (85.37\%) contained both OXA-51 and OXA-23. Additionally, 8 strains $(7.41 \%)$ that contained OXA-58 and $100(92.59 \%)$ strains that contained both OXA-51 and OXA23 were multidrug-resistant. However, 16 (28.07\%) strains containing OXA-51 and 40 (71.43\%) strains containing both OXA-51 and OXA-23 were not multidrug-resistant.

Table 2. PCR results of drug-resistant genes.
\begin{tabular}{lrccrrr}
\hline Genes & Total & $\%$ & Multidrug-resistant & $\%$ & Non-multidrug resistant \\
\hline OXA-51 & 16 & 9.75 & 0 & 0.00 & 16 & 0 \\
OXA-23 & 0 & 0.00 & 0 & 0.00 & 0 & 0 \\
OXA-24 & 0 & 0.00 & 0 & 0.00 & 0.00 \\
OXA-58 & 8 & 4.88 & 8.41 & 92.59 & 40 & 0.00 \\
OXA-51 + OXA-23 & 140 & 85.37 & 100 & & 71.43 \\
\hline
\end{tabular}




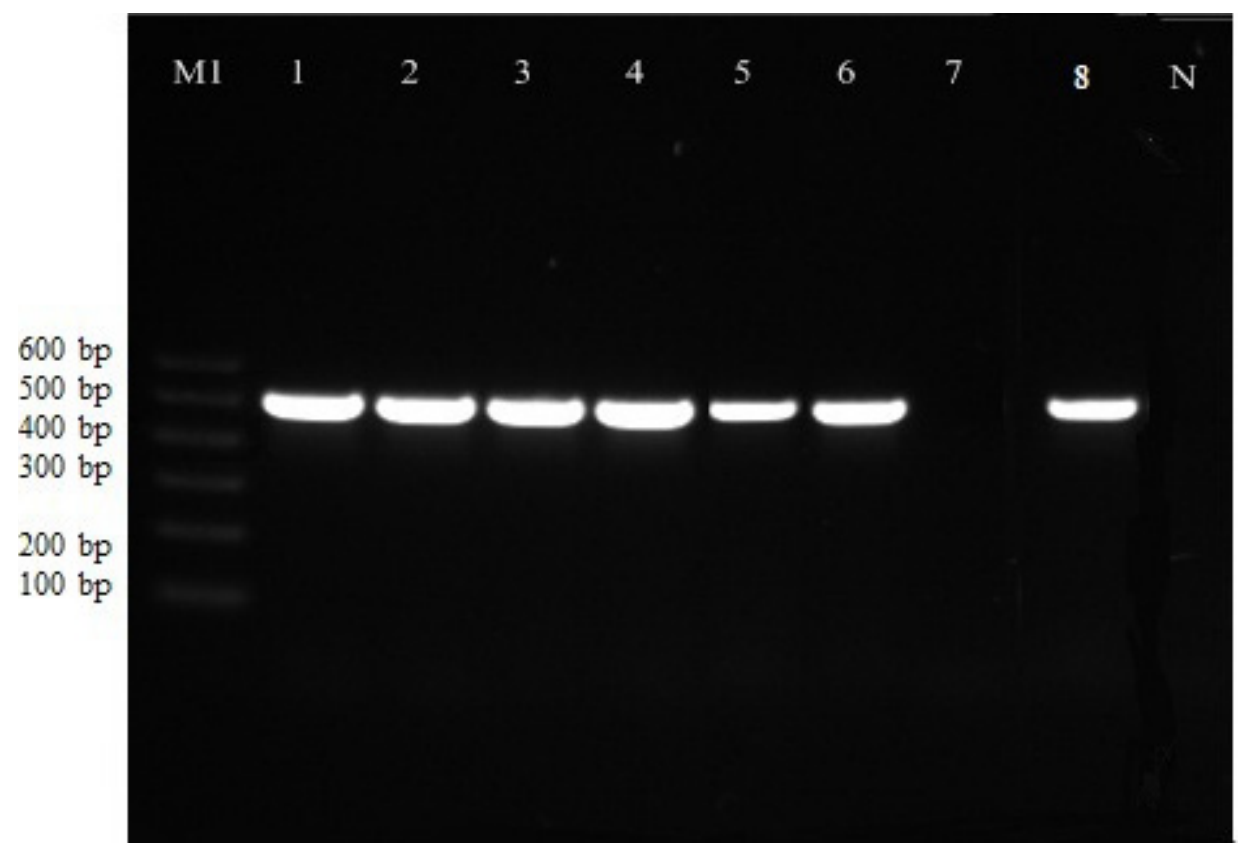

Figure 1. Gene PCR products of OXA-51, OXA-23, OXA-24, and OXA-58. Lane MI = markers; lanes 1- 6,8 = OXA-23; lane $N=$ negative control.

Additionally, we found that $A$. baumannii showed multidrug resistance to penicillin, quinolones, $\beta$-lactam antibiotics, cephalosporin, and carbapenems. Drug resistance rates of $A$. baumannii to amikacin, tobramycin-levofloxacin, and cotrimoxazole were above $90 \%$, and drug resistance rates to ampicillin, cefotetan, cefazolin, cefoperazone, and nitrofurantoin were $100 \%$.

\section{DISCUSSION}

A. baumannii causes a significant number of nosocomial outbreaks worldwide and commonly occurs in settings with high antibiotic selective pressures, such as ICUs. Most outbreak strains are highly resistant to antibiotics, and therefore, therapeutic options are becoming increasingly limited (Turton et al., 2006). We found that the main drug resistance genes were OXA51 and OXA-23 in A. baumannii in isolates from the ICU and pneumology departments.

Our study also shows that the $A$. baumannii is resistant to penicillin, quinolones, $\beta$-lactam antibiotics, cephalosporin, and carbapenems. The main mechanism of drug resistance is due to four carbapenemases. One of the four carbapenemases includes OXA-23, OXA-24, OXA-51, and OXA58. OXA-51 varies between different species of Acinetobacter (Durante-Mangoni and Zarrilli, 2011).

In our study, $16(9.75 \%)$ strains contained OXA-51, 8 (4.88\%) contained OXA-58, and $140(85.37 \%)$ contained both OXA-51 and OXA-23. We did not detect OXA-23 and OXA-24 in any strains. In a recent study, OXA-23 and OXA-58 were detected on bacterial chromosomes, but strains isolated from patients did not contain OXA-24 (Li et al., 2010). Drug resistance rates of $A$. baumannii to amikacin, tobramycin levofloxacin, and cotrimoxazole were above $90 \%$, while drug resistance rates to ampicillin, cefotetan, cefazolin, cefoperazone, and nitrofurantoin were $100 \%$. In 
a previous study, Vakili et al. (2014) reported that $95 \%$ of isolated strains show multidrug resistance and $76.6 \%$ were highly resistant, which is similar to our findings.

We found that OXA-51 and OXA-23 were the main antibiotic resistance genes found in $A$. baumannii isolates, similar to results of previous studies (Mohajeri et al., 2013; Chan et al., 2014; Dettori et al., 2014; Aly et al., 2014). Mohajeri et al. (2013) reported that the OXA-51 and OXA-23 were the predominant mechanisms of resistance to imipenem. Chan et al. (2014) reported that most of the isolated strains in their study contained OXA-23-like and OXA-51-like carbapenemase genes.

In conclusion, we found that isolated strains containing OXA-51 and OXA-23 were more likely to be resistant or have decreased sensitivity to carbapenems. Drug resistance is increasing in $A$. baumannii, and thus, resistance surveillance is becoming increasingly important to prevent the spread of carbapenem-resistant $A$. baumannii.

\section{Conflicts of interest}

The authors declare no conflict of interest.

\section{ACKNOWLEDGMENTS}

We thanks for the help from staffs in our hospital for collection of study samples.

\section{REFERENCES}

Aly M, Tayeb HT, Al Johani SM, Alyamani EJ, et al. (2014). Genetic diversity of OXA-51-like genes among multidrug-resistant Acinetobacter baumannii in Riyadh, Saudi Arabia. Eur. J. Clin. Microbiol. Infect. Dis. 33: 1223-1228.

Cefai C, Richards J, Gould FK and McPeake P (1990). An outbreak of Acinetobacter respiratory tract infection resulting from incomplete disinfection of ventilatory equipment. J. Hosp. Infect. 15: 177-182.

Chan MC, Chiu SK, Hsueh PR, Wang NC, et al. (2014). Risk factors for healthcare-associated extensively drug-resistant Acinetobacter baumannii infections: a case-control study. PLoS One 9: e85973.

Dettori M, Piana A, Deriu MG, Lo Curto P, et al. (2014). Outbreak of multidrug-resistant Acinetobacter baumannii in an intensive care unit. New Microbiol. 37: 185-191.

Durante-Mangoni E and Zarrilli R (2011). Global spread of drug-resistant Acinetobacter baumannii: molecular epidemiology and management of antimicrobial resistance. Future Microbiol. 6: 407-422.

Li C, Wang ZX and Shen JL (2010). Detection of OXA carbapenemases and molecular mechanism in nosocomial outbreak caused by multi-drug resistant Acinetobacter baumannii strains. Chin. J. Clin. Lab. Sci. 28: 383-385.

Lin YC, Sheng WH, Chen YC, Chang SC, et al. (2010). Differences in carbapenem resistance genes among Acinetobacterbaumannii, Acinetobacter genospecies 3 and Acinetobacter genospecies 13TU in Taiwan. Int. J. Antimicrob. Agents 35: 439-443.

Metan G, Sariguzel F, Sumerkan B, Reijden TV, et al. (2013). Clonal diversity and high prevalence of OXA-58 among Acinetobacter baumannii isolates from blood cultures in a tertiary care centre in Turkey. Infect. Genet. Evol. 14: 92-97.

Mohajeri P, Farahani A, Feizabadi MM, Ketabi H, et al. (2013). Antimicrobial susceptibility profiling and genomic diversity of Acinetobacter baumannii isolates: A study in western Iran. Iran J. Microbiol. 5: 195-202.

Petersen A, Guardabassi L, Dalsgaard A and Olsen JE (2000). Class I integrons containing a dhfrl trimethoprim resistance gene cassette in aquatic Acinetobacter spp. FEMS Microbiol. Lett. 182: 73-76.

Poirel L, Pitout JD and Nordmann P (2007). Carbapenemases: molecular diversity and clinical consequences. Future Microbiol. 2: $501-512$

Recchia GD and Hall RM (1995). Gene cassettes: a new class of mobile element. Microbiology 141: 3015-3027.

Sinha M and Srinivasa $\mathrm{H}$ (2007). Mechanisms of resistance to carbapenems in meropenem-resistant Acinetobacter isolates from clinical samples. Ind. J. Med. Microbiol. 25: 121-125.

Srinivasan VB, Rajamohan G, Pancholi P, Stevenson K, et al. (2009). Genetic relatedness and molecular characterization of multidrug resistant Acinetobacter baumannii isolated in central Ohio, USA. Ann. Clin. Microbiol. Antimicrob. 8: 21. 
Sung JY, Kwon KC, Park JW, Kim YS, et al. (2008). Dissemination of IMP-1 and OXA type b-lactamase in carbapenemresistant Acinetobacter baumannii. Korean J. Lab. Med. 28: 16-23.

Turton JF, Ward ME, Woodford N, Kaufmann ME, et al. (2006). The role of ISAba1 in expression of OXA carbapenemase genes in Acinetobacter baumannii. FEMS Microbiol Lett. 258: 72-77.

Vakili B, Fazeli H, Shoaei P, Yaran M, et al. (2014). Detection of colistin sensitivity in clinical isolates of Acinetobacter baumannii in Iran. J. Res. Med. Sci. (Suppl 1): S67-S70. 